\title{
Das Zauberwort heißt Partizipation. Eine Mutrede
}

\author{
Konstanze Marx
}

Die Anfrage, mich mit einem Beitrag an dem vorliegenden Buch zu beteiligen, habe ich gelesen, unmittelbar nachdem sie verschickt worden war. Ich saß gerade im Bundesrat, wo ein Diskussionsforum des Weimarer Dreiecks zum Thema „Fake News, Hate Speech und Bots Herausforderungen für Demokratie und Rechtsstaat?" stattfand.

So kurz vor Jahresende und mit verstohlenem Blick auf die gut gefüllte To-do-Liste für die dem Jahr noch verbleibenden Wochen, sagte ich - Sie ahnen es - trotzdem spontan zu. Das Thema dieses Bandes betrifft mich wie jede Wissenschaftlerin/jeden Wissenschaftler - und wirklich nicht erst seitdem unsere Bundesministerin für Bildung und Forschung Wissenschaftskommunikation in den Fokus der Aufmerksamkeit gerückt hat.

Nebenbei bemerkt: Mit dem in diesem Kontext gern verwendeten Wort „Transfer" ist eigentlich eine Exklusion der Wissenschaft aus der Gesellschaft indiziert, die sich über den Transfer Zugang zurück zur Gesellschaft zu verschaffen sucht; dabei ist sie doch Teil der Gesellschaft und die begriffliche Distanzierung vielleicht sogar kontraproduktiv.

In jedem Fall betrifft mich das Thema sehr, denn die Verknüpfung von Wissenschaft und Gesellschaft sichtbar zu machen, ist mir nicht nur ein Herzensanliegen, ich empfinde es auch als Pflicht - und da unterscheide ich mich nicht von vielen anderen Kolleg*innen -, von meiner Forschung zu

\author{
K. Marx $(\bowtie)$ \\ Universität Greifswald, Greifswald, Deutschland \\ E-Mail: Konstanze.Marx@uni-greifswald.de
}


berichten, die Ergebnisse nicht nur für Peers zu publizieren, sondern auch so, dass Personen sie verstehen, die nicht täglich akademische Texte lesen.

Deshalb habe ich auch getwittert, als ich im Bundesrat saß, hatte also das Smartphone in der Hand, konnte die Anfrage der Herausgeber dieses Bandes lesen und schrieb derweil einen Tweet zur Eröffnungsrede des Regierenden Bürgermeisters von Berlin. Michael Müller betonte, wie wichtig es sei, dass von Hate Speech Betroffene deren Wirkung offenlegen. Damit habe ich einen Aspekt pointiert. Einen weiteren Aspekt konnte ich in meine Timeline projizieren, indem ich den Tweet von @Das_NETTZ retweetete, in dem die Forderung nach digitaler Zivilcourage aus derselben Rede hervorgehoben wurde. Gleichzeitig konnte ich der Initiative Das NETTZ gegenüber Zustimmung, ja Solidarität signalisieren.

Ich konnte also nicht nur über die aktuelle politische Debatte zu einem meiner Forschungsthemen informieren, ich konnte mich zudem auch positionieren. Beides ist von Bedeutung. Warum?

Beginnen wir bei der Positionierung: Vor circa einem Jahr hielt ich in Hamburg im Rahmen des von Jannis Androutsopoulos und Florian Busch ausgerichteten Symposiums „Register des digitalen Schreibens“ einen Vortrag zum Thema „Das Internet macht die Sprache kaputt.“ Ein linguistischer Zugang zu metasprachlichen Reflexionen von ,Laien'. Ich zeigte unter anderem, wie der Forschungsgegenstand Hate Speech ethnomethodologisch konturiert wird - man könnte es auch so ausdrücken: wie der Gegenstand, der zweifelsohne auch eines sprachwissenschaftlich fundierten Zugangs bedarf, schneller und sichtbarer (natürlich auch oberflächlicher) von anderen (nicht-wissenschaftlichen Institutionen) aufgegriffen und bearbeitet wird.

Sei es durch ein Erklärvideo der Tagesschau, das seit Mai 2017 online verfügbar ist, sei es durch die auf der Facebook-Seite von Renate Künast zusammengetragenen Charakteristika von Hass-Kommentaren im „HassTool“, sei es durch populärwissenschaftliche Fernsehformate - sogenannte Social Factuals - wie „Manipuliert. Mit Sascha Lobo“ bei ZDFneo. Was in vergleichbarer Präsenz fehlte, war meines Erachtens der Beitrag der Linguistik. In der Linguistik (und auch in anderen Wissenschaftsdisziplinen) gab es schon Theorien zu verbaler Gewalt, als an das Social Web noch gar nicht zu denken war. Es stand also die Frage im Raum, wie sichtbar eigentlich ist, was die Linguistik (zum Thema) leistet. Das führte mich zunächst $\mathrm{zu}$ der Frage, wie eigentlich über mein Arbeitsgebiet und Kolleg*innen in traditionellen Medien verhandelt wird. Drei Beispiele möchte ich diesbezüglich aufrufen: 
1. In einem Radiointerview, das eine Journalistin des SWR 2 im November 2016 mit der Mannheimer Sprachwissenschaftlerin Eva Gredel führte, lässt sich anhand der Fragetechnik nachvollziehen, dass in der Wissenschaftlerin eher eine Statistin gesehen wird: Sie soll lediglich die eigenen Vorannahmen bestätigen. Diese sind im Wesentlichen: Die Sprache verrohe und niemand sei mehr an einem direkten Gespräch interessiert.

2. Am 22. Oktober 2016 erscheint in der FAZ eine Rezension des Buches „Schreiben digital. Wie das Internet unsere Alltagskommunikation verändert" von Christa Dürscheid und Karina Frick. Der Autor - Wolfgang Krischke - kritisiert hier ein sogenanntes „Linguisten-Mantra“, das etwa laute: Jeder Sprachwandel sei automatisch ein Fortschritt, weil er ja den Bedürfnissen der Sprecher entspringe.

3. Im Januar 2018 schreibt derselbe Journalist in einer Kolumne dem inzwischen emeritierten aber deswegen nicht minder aktiven Angewandten Linguisten Gerd Antos, Bezug nehmend auf seinen Aufsatz „Wenn Roboter „mitreden“ ... Brauchen wir eine Disruptions-Forschung in der Linguistik?“" zu, sich der „romantischen“ Vorstellung hinzugeben, die Essenz menschlicher Kommunikation sei der persönliche Dialog. Hier wurde das Bild der weltfremden, verträumten und auf der Stelle tretenden - denken Sie an die Mantra-Assoziation - Sprachwissenschaft gezeichnet, ein ungerechtfertigter Vorwurf, dem zu widersprechen ist.

Aber ich hatte das Glück, dass der letztgenannte Journalist im Publikum saß. Er meldete sich zu Wort und bemerkte, sein wichtigster Kritikpunkt sei, dass bei all unserer Arbeit die eindeutige Positionierung fehle. Immer würden wir abwägen, auf die Verwendung von Sprache in spezifischen Kontexten verweisen, von uns kämen keine klaren Aussagen.

Das mag daran liegen, dass sich der Forschungsgegenstand Sprache der Fixierung entzieht: Sprache lebt, ist dynamisch. Das auszuhalten fällt mitunter nicht leicht. Dass Sprachverwendung viele Facetten vereint, weder als richtig noch als falsch zu beurteilen ist, muss einen jedoch nicht in die Verzweiflung und schon gar nicht in Zynismus treiben. Es gibt andere Parameter, die einer Bewertung zugrunde liegen können, etwa - wie von Jürgen Schiewe empfohlen - die Angemessenheit. Anatol Stefanowitsch schlägt gar Moral als Gradmesser vor. Das soll an dieser Stelle jedoch gar nicht vertieft werden. Es führt uns lediglich zurück an den oben bereits erwähnten Punkt der Positionierung, die mir ein Medium wie Twitter durchaus ermöglicht, eine wissenschaftliche Publikation jedoch nur bedingt, weil meine Meinung 
zu einem Analyseresultat sekundär ist. Oder andersherum: Twitter zwingt es einem gewissermaßen auf, das Format „erquengelt“ sich quasi einen Standpunkt.

Viele Wissenschaftler*innen schreckt das ab. Vielleicht ahnt die Historikerin Charlotte Jahnz (@Cjahnz) das, wenn sie am 15. Oktober 2018 auf Twitter zu einer „Umfrage, die ich gerne mal lesen würde: den Großteil der deutschen Professorenschaft gesammelt fragen, warum er nicht twittert. Reichen auch fünf Sätze für jeden“ animiert. Anna-Lena Scholz (@doc_scholz) macht daraufhin die Frage „Warum twittern Sie nicht?" in einem „Chancen Brief" der ZEIT zum Thema.

Das Unvermögen, sich kurz zu fassen, und die Skepsis, dass sich komplexe Sachverhalte über Twitter vermitteln lassen, werden als Gründe genannt. Im oben genannten Zusammenhang kann hier bereits vermutet werden, dass das wohl gar nicht die Erwartung an twitternde Wissenschaftler*innen zu sein scheint. Die Möglichkeiten jedoch, Threads zu verfassen, mit Artikeln zu verlinken oder die 280 Zeichen (interessanterweise hält sich bei Kritiker*innen hartnäckig das Gerücht, auf Twitter dürfe man immer noch nur 140 Zeichen schreiben) auszuschöpfen, sind darüber hinaus einen Versuch wert.

Es gibt Nicht-Nutzer*innen, die als Grund fürs Nicht-Twittern anführen, sich für langsam zu halten - hier möchte man einwenden, woran das zu messen wäre. Und selbst wenn vergleichbarer Inhalt bereits gepostet wurde, weil man gerade die gleiche Konferenz und den gleichen Vortrag besucht, zeigt das doch nur, dass auch andere Kolleg*innen etwas als relevant erachten, dem man selbst auch Bedeutsamkeit zumisst und es deshalb zum Inhalt eines Tweets macht. Man kann in solchen Momenten aufeinander verweisen und die Stimme, mit der man dann gemeinsam spricht, ist vielleicht sogar ein wenig „lauter“.

Andere möchten keine unnötigen Datenspuren ins Netz streuen. Wieder andere halten Twitter für unseriös, was ungefähr so unüberlegt ist, wie das Pauschalurteil der Staatsministerin für Digitales, Dorothee Bär, die in einem Interview mit der Welt am 10. März 2018 behauptete: „Auf Twitter sind ohnehin nur Politiker, Journalisten und Psychopathen unterwegs.“

Auch der Einwand, Twitter könne persönliche Kommunikation nicht ersetzen, ist so überzeugend wie der Einwand, dass man Konzerte meiden sollte, weil man kein Duett mit den Interpret*innen auf der Bühne singen dürfe. Wobei - bei Twitter bekäme man vielleicht sogar die Gelegenheit dazu, möglich ist es, vorrangiges Ziel vielleicht eher nicht.

Manche Nicht-Twitter*innen halten sich selbst nicht für wichtig genug, führen mangelnde „Selbstdarstellungstendenzen“ an und die Überzeugung, 
kein „Megastar“ zu sein - ein interessanter Ansatzpunkt, der eigentlich aber rückwirkt auf die potenziellen Rezipient*innen, die dann uninformiert bleiben.

Und so fragt Anna-Lena Scholz verständlicherweise eine Woche nach Abschluss der Umfrage: „Sind WissenschaftlerInnen, die twittern, wirklich selbstdarstellerisch? Oder nehmen sie vielleicht ihre Arbeit ernst? Wieso glauben Wissenschaftler, sie hätten nichts zu sagen? Wer ist denn der Adressat, den sie ernst genug nehmen, dass er oder sie ihre Aufmerksamkeit verdient?"

Darüber hinaus scheint die Einschätzung, nicht wichtig genug zu sein, angesichts der Vielzahl an Twitter-Nutzer*innen überwindbar und motiviert gleichzeitig dazu, den Fokus weg von der eigenen Person hin zur Forschung $\mathrm{zu}$ verschieben. Es geht vielen Wissenschaftler*innen auf Twitter einfach darum, die Kolleg*innen auf dem Laufenden zu halten, sich gegenseitig mit Literaturhinweisen zu versorgen, Methoden zu vergleichen, auf Publikationen hinzuweisen - das Gespräch am Rande von Konferenzen weiterzuführen, obwohl die Konferenz längst vorbei ist und die nächste noch nicht in Sicht. Und es funktioniert auch in die andere Richtung: Man trifft sich zwar auf Konferenzen zum ersten Mal, kennt sich aber schon von Twitter - ein guter Anlass, persönlich ins Gespräch zu kommen. Natürlich signalisiert man auch Journalist*innen und jeder anderen interessierten Person, ansprechbar zu sein, somit ein Mensch, der einem bei Bedarf Rede und Antwort stehen und fachliche Fragen beantworten würde.

Damit ist mit einem weiteren Vorurteil gegenüber Sozialen-NetzwerkSeiten aufzuräumen: der von Kritiker*innen skizzierten anonymen, unidirektionalen One-to-many-Kommunikation. Nur wenige der im deutschsprachigen Raum twitternden (Sprach-)Wissenschaftler*innen haben einen „Fame-Status“ erreicht, der die Befürchtung rechtfertigen würde, eine direkte Adressierung drohe im digitalen Nirvana zu dissoziieren.

Dass es das wiederum gibt: Sprachwissenschaftler*innen mit - ich nenne es einmal zeitgemäß - „Influencer“-Renommee außerhalb der wissenschaftlichen Community - wer hätte das für möglich gehalten? Gerade in einer Zeit, in der das gesellschaftliche Ansehen der Geisteswissenschaften leidet, wie Maximilian Günnewig-Mönert am 22. Mai 2017 auf prisma-hsg.ch feststellt, gerade in dieser Zeit ist es zu begrüßen, wenn es Geisteswissenschaftler*innen und aus meiner Sicht natürlich insbesondere Linguist*innen gelingt, breites Interesse und Aufmerksamkeit zu binden. Schließlich sind „weder [...] ihre Gegenstände obsolet noch ihre Methoden desavouiert“, wie es Walter Schweidler (2007, S. 352) ausdrückt.

Selbst wenn das die wenigen Ausnahmen sind, die Philippe Wampfler (@phwampfler) meint, wenn er am 29. Oktober 2018 tweetet „,...] 
Wissenschaftler*innen können bis auf Ausnahmen fast nur verlieren, wenn sie in Massenmedien präsent sind."

Sein Punkt ist natürlich klar. Auch ich muss Ergebnisse meiner Arbeit für die Darstellung in Presse- oder Rundfunk-Interviews „entschlacken“ und kann Zusammenhänge vielleicht nicht in gebührender Komplexität darstellen. Möglicherweise schlägt mir dafür in der eigenen Community nicht nur Wohlwollen entgegen - man weiß es ja nicht. Dennoch möchte ich Wampflers defätistische Prognose nicht teilen. Die Synergieeffekte, die Fachpublikationen, populärwissenschaftliche Veröffentlichungen und die Präsenz auf Twitter haben, sind meines Erachtens ein positives Signal im Hinblick auf die Sichtbarkeit des Fachs; und das ist natürlich nicht zu beschränken auf die Linguistik. Letztlich - so glaube ich zu erahnen - geht auch Philipp Wampfler das Risiko weiter ein, orientiert an seiner so wichtigen Botschaft, der Verknüpfung digitaler Methoden mit dem Deutschunterricht.

Abgesehen davon hatte ich persönlich aber auch gar keine Wahl: Ich beschäftige mich mit der Sprachverwendung und der Kommunikation im Social Web, wie könnte ich mit diesem Forschungsinteresse dem Kommunikationsraum fernbleiben? Man kann Interaktion auf den verschiedenen Plattformen kaum untersuchen, wenn man nicht partizipiert und die plattforminhärenten Regeln identifiziert - davon bin ich überzeugt. Die jeweilige Sprach- und Kommunikationskultur wird von den Teilnehmenden tradiert, thematisiert und aktiv ausgehandelt. Nur wenn man selbst auch schreibt, Kommentare er- und aushält, spezifische Formulierungen erlernt, Konventionen detektiert und Akzeptanz (oder auch Widerspruch) durch Anschlusskommunikation erfährt, kann Wissen über plattformentypische Prozesse generiert werden.

Gleichzeitig erhält man einen qualitativen Zugang zum sprachlichen Material, den eine quantitative - auf Masse ausgerichtete - Datenerhebung nicht gewähren kann. Bei der Archivierung von Webcrawling-Resultaten werden durch abweichende Darstellungsformate (etwa in Listen) für die Analyse relevante Oberflächenphänomene modifiziert oder getilgt - etwa die Anordnung der Nachrichten, die Identifizierbarkeit von Initialposts, Antworten darauf oder Antworten innerhalb eines Threads, die Verwendung von Emojis, der Einsatz von Memes - was für die qualitative Analyse insgesamt nachteilig ist.

Gleichzeitig lässt sich Forschungsmaterial zu Verbalgewalt ohnehin nicht problemlos automatisch detektieren (auch wenn es Versuche, wie die von Sylvia Jaki und Tom DeSmedt gibt): Online-Gewalt oder Hate Speech erschöpft sich nicht im Gebrauch von Schimpfwörtern, sondern 
nimmt viele Gestalten an - bis hin zur „Übernahme von unverdächtigen, ja gesellschaftlich hochkonsensuellen Werten“ und der „Lexik von Gruppen, die dem Verdacht rassistisch zu sein, wenig ausgesetzt ist", wie es Christian Bendl und Jürgen Spitzmüller (2017) formulieren. Wir finden hier wieder, was in der linguistischen Sprachkritik als „Kampf um Begriffe“ bezeichnet wird, etwa von Armin Burghardt (2011). Man muss die sprachlichen Belege also lesen und in ihren aktuellen Kontexten evaluieren. Das setzt den Zugang zu diesen Kontexten voraus und diesen erhalte ich, indem ich mich selbst auf die Plattformen begebe: teilnehmende Beobachtung 2.0.

Ein weiterer wichtiger Aspekt, von dem ich nur durch Partizipation in sozialen Netzwerken weiß, ist: Von Online-Gewalt betroffene Personen republizieren das Datenmaterial - z. B. als Bild (also in einem für Webtext-Crawler nicht lesbarem Format). Als Beispiel, das einen gewissen Bekanntheitsgrad erreicht hat, sei der an Dunja Hayali gerichtete Hassbrief von „Lawrence von Arabien“ genannt, den die Journalistin sowohl auf Twitter als auch auf Facebook veröffentlicht hat. Es gibt eine ganze Reihe derartiger Republikationen, die eine Datenquelle darstellen, die selbst bei auf Suchbegriffen (z. B. Schimpfwörter) beschränktem Datencrawling unentdeckt bliebe. Weiterhin ergeben sich dadurch Kommunikate, die ganzheitlich betrachtet werden müssen. So ist es interessant, wie sich die betroffene Person zu der Verbal-Attacke positioniert, wie sich die Anschlusskommunikation gestaltet usw.

Als vielzitiertes Phänomen der Gesellschaft 2.0 muss Hate Speech auch als Interaktionsphänomen betrachtet werden. Auf Plattformen kommt es nicht nur zu Angriffen, sondern auch zu Verteidigung, zu Solidarisierung auf der einen wie auf der anderen Seite, das Phänomen selbst wird verhandelt und ethnomethodologisch konturiert (ich habe es oben bereits erwähnt). Man kann beobachten, wie sich verschiedene Formen der sogenannten Counter Speech, der Gegenrede, herausbilden, welche Strategien hierbei zur Deeskalation oder zur Eskalation führen. Die Rekontextualisierung von Hate Speech, Cybermobbing oder auch Cybergrooming gibt Aufschluss über Strategien der diskursiven Bearbeitung von Verrohung und deren Wahrnehmung und über deren Erfolg oder Misserfolg, was für die Prävention unverzichtbar ist.

All das fließt in die Betrachtung des Gegenstands ein und deshalb ist es hilfreich, Facebook-Profile aufmerksam zu lesen, Twitter-Debatten $\mathrm{zu}$ verfolgen und sogar auch in Online-Spielen aktiv zu sein, in deren Interaktionssträngen die Kommunikationskultur des Social Web auf deren Extreme reduziert projiziert wird. Cybergrooming, Extremismus, Volksverhetzung - all das findet sich in Spielen, die teilweise keiner 
Altersbeschränkung unterliegen. Wenn ich darüber nichts weiß, aber mit Personen auf Twitter vernetzt bin, die sich in diesen Bereichen bewegen, erhalte ich wichtige Hinweise und kann mein Forschungsfeld entsprechend ausweiten.

Auch die insbesondere auf Twitter viel zitierte und satirisch gerahmte Whats-App-Gruppenkommunikation erweist sich im großen Kontext der linguistischen Konfliktforschung ergiebig: Konflikte bahnen sich an, entstehen aber auch aufgrund von der jeweiligen Plattform eigenen Affordanzen, die - und auch das wird auf Twitter offenbar - von den Nutzer*innen selbst kritisch reflektiert werden.

Schließlich können auf diese Weise gewonnene Analyseergebnisse in Handlungsanweisungen münden, die systematisch in den Kommunikationsraum zurückgegeben werden können. In meinem Fall gibt es also gute Gründe, sich in das „digitale Getümmel“ zu stürzen.

Andere halten es - und das führt mich zurück zur Umfrage von AnnaLena Scholz - für Zeitverschwendung und sehen keinerlei Mehrwert darin. Das kann daran liegen, dass sie es noch nicht ausprobiert haben. Angesichts des Zeitaufwands, der mit Twitter verbunden ist (auch das wird übrigens als Grund genannt, der Plattform fernzubleiben), ist das nachvollziehbar, obwohl man einwenden möchte, dass auch Twitter-Aktivität mithilfe des Tweetdecks beispielsweise effizient organisiert werden kann. Und natürlich ist insgesamt die Entscheidung einer jeden Wissenschaftlerin/eines jeden Wissenschaftlers gegen Twitter zu tolerieren. Insbesondere dann, wenn sich - wie eine Antwort aus der Umfrage offenbart - Frust breitmacht, weil der eigene Account eine Angriffsfläche bietet. Das ist zum Beispiel nachweislich und überdurchschnittlich häufig bei Forscher*innen der Fall, die sich den Gender-Studies widmen. Vor diesem Hintergrund ist auch Christina Hölzels (@ChristinaHolzel) Tweet vom 22. November 2018 zu verstehen: „Gewöhne mich daran, dass die CAU nicht auf twitter ankündigt, was ich relevant fände. Dabei wäre, der Online-Alltag als Feministin ' doch wirklich ein digital vermarktbares Thema. Vermute Vor-, nicht böse Absicht. Freue mich so oder so auf Jasna Strick und Andreas Kemper. \#gender." Aber - das zeigt eine der Antworten in der Umfrage - man muss nicht Genderforscher*in sein: „gefühlten $95 \%$ der Internetnutzer [bietet die Tatsache,] Ingenieurin, junge Professorin, Wissenschaftlerin, arbeitende Mutter, Politikinteressierte, Feministin [zu sein,] leicht hebbares Kritikpotenzial an [der] Person."

Anna-Lena Scholz kategorisiert in ihrer Antwort auf den Einwand, man habe die „Hexenjagden“ nicht mehr ertragen, Shitstorms als „Randphänomen, das sich ausblenden lässt.“ Das lässt sich vielleicht dann einfach 
sagen, wenn man noch nie von Attacken betroffen war oder sich inzwischen ein sprichwörtlich „dickes Fell“ zugelegt hat. Zwischen diesen Polen stehen aber Schockerfahrungen, tiefe Verletzung, Verunsicherung, ja wahrscheinlich auch Angst - selbst wenn Shitstorms ihrem diskursiv zugewiesenen Namen selten entsprechen. Es reicht letztlich ein einziger aggressiver, despektierlicher und grenzverletzender Kommentar, um einen Menschen zu verstören, ihm/ihr emotionalen Schmerz zuzufügen.

Deshalb erachte ich es ja als so wichtiges Forschungsfeld, deshalb sollen ja Wege in einen höflich-rücksichtsvollen Diskurs gefunden werden - ein Diskurs, aus dem sich niemand ausklinken muss, weil er/sie diskreditiert wird. Beteiligung ist mehr als erwünscht und notwendig. Don't feed the Troll das war gestern. Wir wissen heute, dass es Sinn - im Sinne von Aussicht auf Erfolg - hat, zivilisierte Diskurspraktiken zu verteidigen, und sollten einen so wertvollen Interaktionsraum nicht Chaot*innen überlassen, denen es einzig und allein darum geht, andere zu provozieren. Schließlich wird auch ihr Vorgehen so einsehbar und es können Strategien entwickelt werden, wie diesem Verhalten begegnet werden kann.

Letztlich muss man zudem sagen, dass die, die sich nicht (mehr) beteiligen, auch etwas verpassen.

Twitter ist ein Forum, in dem wichtige gesellschaftliche und auch wissenschaftspolitische Debatten angestoßen und geführt werden. Ich greife einmal die Hashtags \#metoo, \#unten oder \#bildungsstreik als Beispiele heraus. So spiegelt die Plattform, auf der es zweifelsohne Diskriminierung gibt, diese Diskriminierung eben auch wider und rückt Problematiken in ein kollektives Bewusstsein, die vielleicht nie zur Sprache gekommen wären.

Twitter ist ein Forum, in dem Menschen einander unterstützen (siehe etwa \#EineSorgeWeniger), Kindern Weihnachtswünsche erfüllen, wenn ihre Eltern es sich nicht leisten können, in dem Alleinerziehende eine Stimme erhalten haben und inzwischen gehört werden - auch „außerhalb“ der Plattform (Christine Finke, @Mama_arbeitet), in dem sich Menschen beim Sterben begleiten lassen und aufrichtig betrauert werden, auch von Menschen, die die Sterbenden nie persönlich getroffen haben.

Twitter ist offenbar ein Forum, das stadtfräulein (@Schaumdompteur) zufolge Mut zur Menschlichkeit gibt; sie twittert am 7. Oktober 2018: „Was ich mich ohne Twitter nie getraut hätte oder überhaupt auf die Idee gekommen wäre: Mich ganz selbstverständlich zwischen pöbelnde Weiße und einen „Nicht-Deutschen“ stellen. Und schon stand eine weitere Frau neben mir, wir lächelten uns zaghaft an - Ruhe war. \#keinortfürnazis“.

Twitter ist ein Forum, in dem die Grenzen der Sagbarkeit in der oben angesprochenen Dimension überstrapaziert, aber im Hinblick auf Tabus oder 
Themen, über die es zu sprechen schwerfällt, erweitert werden: die Pflege eines schwerstkranken Kindes etwa (22 Monate, @22Monate), Krebsleiden (Metahasenbändigerin (Onkobitch), @dieKali) oder das Leben nach dem Verlust eines geliebten Menschen (Weg ins Leben 2.0, @Cancer_is_an_ah).

Mit Blick auf die Wissenschaftskommunikation ist Twitter ein Forum, das Einblicke in den Arbeitsalltag von Forscher*innen gewährt (@realscientists), aktuelle Forschungsthemen vorstellt, Wissenschaft als lebendiges Feld abbildet und die Mär vom Elfenbeinturm, quasi sekündlich, ad absurdum führt. Damit öffnet sich nicht nur der Blick auf inspirierende Methoden und spannende Ergebnisse, sondern auch auf die prekären Bedingungen, unter denen in Deutschland geforscht wird, geforscht werden muss - insbesondere im akademischen Mittelbau. Als Beispiel sei hier der Twitter-Thread von Kilian genannt, der am 4. Dezember 2018 auf dem von wöchentlich wechselnden Autor*innen gestalteten Account @realsci_DE (Real Scientists) postete: „Aus gegebenem Anlass ein paar Gedanken zum Arbeiten in der Wissenschaft und prekären Arbeitsverhältnissen in derselben. Ich selbst habe vor meiner jetzigen 5-Jahres-Stelle drei Jobs in drei verschiedenen Ländern gehabt." Somit ist Twitter im ursprünglichen Sinne ein Medium, das Transparenz ermöglicht und auf andere Orte der Transparenz verweist: Orte, an denen neue Formen der Wissenschaftskommunikation praktiziert werden, wenn etwa Lambert Heller (@Lambo) fragt: „Wie viele von den Direktor*innen der 93 Institute der @LeibnizWGL bloggen eigentlich? Henning Lobin jedenfalls bloggt als Direktor des IDS (weiter) bei den @scilogs, zuletzt über seine Mitarbeit im Rechtschreibrat - und das Gender-Sternchen. cc @textundblog @IlonaRiek“, aber auch wenn die Blogger*innen selbst auf Twitter sind, siehe @HenningLobin (Engelbert-Galaxis), @AlexanderLasch (Sprachpunkt), Simon Meier @fussballinguist (Fußballlinguistik) oder Kristin Kopf (@Schplock) und Anatol Stefanowitsch (@astefanowitsch; Sprachlog).

Aber es sind ja nicht nur Blogs, wie von Annette Leßmöllman (@annetteless) am 7. Dezember 2018 zu erfahren war: „Raus aus den Echokammern“ \#Wisskomm für ein breites Publikum, inkl. unser Projekt \#wissfueralle in der @tazgezwitscher von@ManRonzheimer taz.de/!5551542/ @schroep @cml_net": In dem verlinkten Zeitungsartikel wird das erfolgreiche Projekt „Haus der kleinen Forscher" beschrieben, aber eben auch ein neues am Karlsruher Institut für Technologie (KIT) angesiedeltes Projekt mit dem Namen „Wissenschaft für alle“.

Und auch das von Mark Dang-Anh, Matthias Meiler und mir gegründete Journal für Medienlinguistik, in dem wir den Weg eines wissenschaftlichen Artikels von der Einreichung über das Review-Verfahren bis zur Publikation 
transparent machen und für den fachlichen und nicht-fachlichen Diskurs öffnen, verfügt über seinen eigenen Twitter-Account (@jfml_journal).

Bei Twitter kreuzen sich die Wege, können umgelenkt werden, auseinander und an anderer Stelle wieder zusammenführen, Umwege werden oder direkte Verbindungen, das Potenzial ist - wie ich hoffentlich zeigen konnte - groß. Und vielleicht kann ich diesen Gedanken unterstützend hinzufügen, dass es in der eingangs benannten Veranstaltung im Bundesrat zwar einen - wie Thomas Wüst (@Thomas_Wuest) twittert „Bedrückende[n] Moment bei \#DemokratieInsNetz-Diskussion im @ bundesrat: [gab] @SawsanChebli konstatiert ,Ich erlebe fast täglich \#shitstorms' und hat gestern ihren \#Facebook-Account deaktiviert, weil sie die Vielzahl der \#Shitstorms nicht mehr monitoren konnte [Tränen-Emoji].“ Es handelt sich hier um eine Entscheidung, die verständlich, aber aus vielen Gründen zu bedauern ist. Mut - in dieser Situation - macht jedoch, dass ihr privater Twitter-Account nach wie vor aktiv ist.

\section{Literatur}

Bendl, C., \& Spitzmüller, J. (2017). ,Rassismus' ohne Rassismus? Ethnoseparatistische Diskurse in sozialen Netzwerken. Wiener Linguistische Gazette, 26, $1-26$.

Burkhardt, A. (2011). Linguistisch begründetes Missvergnügen. Über systembezogene und polit(olinguist)ische Sprachkritik. In J. Schiewe (Hrsg.), Sprachkritik und Sprachkultur. Konzepte und Impulse für Wissenschaft und Öffentlichkeit (S. 97-123). Bremen: Hempen.

Schweidler, W. (2007). Den eigenen Gegenständen gerecht werden. In J.-D. Gauger \& G. Rüther (Hrsg.), Warum die Geisteswissenschaften Zukunft haben! - Ein Beitrag zum Wissenschaftsjahr 2007 (S. 344-357). Freiburg i. Br.: Herder.

Konstanze Marx ist Lehrstuhlinhaberin für Germanistische Sprachwissenschaft an der Universität Greifswald. Sie wurde mit einer neurolinguistischen Arbeit zum Textverstehen an der Friedrich-Schiller-Universität Jena promoviert und mit einer Arbeit zum „Diskursphänomen Cybermobbing. Ein internetlinguistischer Zugang zu [digitaler] Gewalt" (2017 bei de Gruyter erschienen) an der Technischen Universität Berlin habilitiert. Im Anschluss arbeitete sie als Professorin für die Linguistik des Deutschen an der Abteilung Pragmatik des Leibniz-Instituts für Deutsche Sprache Mannheim und an der Universität Mannheim. Ihre Schwerpunkte liegen in der Internetlinguistik, der Diskurs- und Textlinguistik, der Erforschung des Zusammenhangs zwischen Sprache-Kognition-Emotion, der Genderlinguistik und der medienlinguistischen Prävention. 
Open Access Dieses Kapitel wird unter der Creative Commons Namensnennung 4.0 International Lizenz (http://creativecommons.org/licenses/by/4.0/deed.de) veröffentlicht, welche die Nutzung, Vervielfältigung, Bearbeitung, Verbreitung und Wiedergabe in jeglichem Medium und Format erlaubt, sofern Sie den/die ursprünglichen Autor(en) und die Quelle ordnungsgemäß nennen, einen Link zur Creative Commons Lizenz beifügen und angeben, ob Änderungen vorgenommen wurden.

Die in diesem Kapitel enthaltenen Bilder und sonstiges Drittmaterial unterliegen ebenfalls der genannten Creative Commons Lizenz, sofern sich aus der Abbildungslegende nichts anderes ergibt. Sofern das betreffende Material nicht unter der genannten Creative Commons Lizenz steht und die betreffende Handlung nicht nach gesetzlichen Vorschriften erlaubt ist, ist für die oben aufgeführten Weiterverwendungen des Materials die Einwilligung des jeweiligen Rechteinhabers einzuholen. 